\title{
Effect of Injection Rate and Post-Fill Cure Pressure on Properties of Resin Transfer Molded Disks
}

\author{
Kristian A. Olivero, ${ }^{1}$ Harry J. BArrazA, ${ }^{2}$ Edgar A. O'ReAR ${ }^{2}$ \\ AND M. CENGIZ ALTAN ${ }^{1, *}$ \\ ${ }^{I}$ School of Aerospace and Mechanical Engineering \\ ${ }^{2}$ School of Chemical Engineering and Material Science \\ University of Oklahoma \\ Norman, Oklahoma, 73019, USA \\ (Received October 6, 2001) \\ (Revised March 5, 2002)
}

\begin{abstract}
The effects of flow rate and post-fill cure pressure, i.e., packing pressure, on the mechanical properties of resin transfer molded disks are experimentally investigated. An experimental molding setup is constructed to fabricate fiber-reinforced, center-gated, disk-shaped composite parts. Disks are molded at different flow rates and packing pressures in order to observe the effects of these parameters on the mechanical properties and void content of the final parts. Specimens are cut from three different locations in the molded disks for testing. Specimens from the first two locations are tensile tested to obtain strength and stiffness properties, and the third location is used for microscopic analysis to determine void content and void properties. Increased injection rate is found to reduce both the strength and stiffness of the molded parts due to more voids induced by the faster moving fluid front. Packing pressure is also found to have a significant effect on specimen properties. At higher packing pressures fewer voids and improved strength and stiffness are observed. Mechanical properties are correlated with total void fraction for disks molded at different packing pressures. Exponential decrease in both tensile strength and elastic modulus is observed with increasing void fraction. Doubling the void volume fraction from 0.35 to $0.72 \%$ results in a $15 \%$ decrease in strength and a $14 \%$ decrease in stiffness. The results demonstrate that selection of suitable injection rate and addition of packing pressure to resin transfer molding (RTM) process can improve mechanical properties of molded parts considerably.
\end{abstract}

KEY WORDS: resin transfer molding, packing pressure, voids, injection rate.

\footnotetext{
*Author to whom correspondence should be addressed. E-mail: altan@ou.edu
} 


\section{INTRODUCTION}

\section{Resin Transfer Molding}

$\mathbf{R}$ ESIN TRANSFER MOLDING (RTM) is frequently used to fabricate medium to large, geometrically complex composite parts in a cost effective manner. In this process, a fibrous reinforcing preform is placed in the mold cavity and subsequently impregnated by a relatively low viscosity thermosetting resin. Compared to other molding operations that use higher viscosity thermoplastics, the RTM process can be accomplished with much simpler, lighter, and less expensive tooling and molding equipment. RTM has become increasingly popular because it offers improved properties at a lower cost. Overall cost reduction is primarily due to increased production rates a result of automation, low capital cost, and low scrap rate [1].

\section{Importance of Process Parameters}

Several process parameters are important to the properties and quality of RTM parts. Process parameters influence phenomena during molding such as wetting, air entrapment, and thermal gradients. Poor wetting and air entrapment during molding lead to voids that degrade mechanical properties and surface finish in the final part, while thermal gradients can cause the part to deform or buckle as it cures [2]. Parameters such as injection rate [3-5], gate and vent locations, molding or packing pressure [6-8], and preform microstructure [9-11] are commonly known to contribute to void formation. Quantitative details about how individual process parameters lead to void formation, and the resulting change in mechanical performance are vital to understanding and design of effective RTM processes.

A number of mechanical testing methods are utilized in the literature to measure the performance of RTM parts fabricated using varied process parameters. Callus et al. examined molded parts containing 60\% glass fibers impregnated by Derakane 411-c50 vinyl ester resin for several fiber weave architectures [12]. Parts were tensile tested in both the warp and weft directions. Three distinct regions were observed in the stress-strain curves - an elastic regime, a hardening phase, and softening phase. Mechanical testing was complimented by optical microscopy, in which they observed resin between tows failing at low strains, although this did not reduce the elastic modulus. De Oliveira Simões and Marques obtained the stiffness properties of glass and hybrid carbon-glass fiber reinforced epoxy resin [13]. Stiffness properties were determined from the axial and circumferential strains recorded during internal water pressure tests of tubular specimens. Hillermeier and Seferis investigated a method of improving the toughness of RTM parts by manufacturing interlayered RTM composite structures [14]. They performed several tests on the molded parts to detect improved toughness by dynamic mechanical analysis (DMA), double cantilever beam (DCB) method for mode I interlaminar fracture toughness, and an end notch flexure (ENF) test to measure mode II interlaminar fracture toughness. Margueres et al. compared ultrasonic testing to several stiffness and damage measurement techniques, including tension, torsion, impact, fatigue, and post-impact fatigue [15]. For undamaged samples all of the methods were shown to be adequate. Their results also showed the capability of non-destructive methods in qualitatively and quantitatively describing the behavior of a damaged material. Most of the studies on mechanical 
characterization of RTM parts focus on the effect of the reinforcement type and properties such as the weave pattern. Studies attempting to correlate mechanical properties with the process parameters such as injection rate and packing pressure are limited [3-5].

\section{Importance of Void Content}

One of the main effects of varying the process parameters in RTM is changing void occurrence and characteristics in the molded parts. Microvoids are common defects formed in the liquid molding of composite materials due to the flow dynamics and the interactions between the resin and the fiber surfaces inside the mold cavity, and are the major contributor to mechanical degradation of RTM parts. Voids occur at different length scales, such as the fiber, tow, and part scales, with different factors contributing to formation of voids at each scale. A composite part having a high void content usually exhibits lower structural integrity, in terms of tensile strength and interlaminar shear strength (ILSS) [16-18]; as well as an increased propensity to absorb moisture from the environment [19]. Crack propagation and failure mechanisms have also been shown to correlate, not only with void content, but also with the void size and shape distribution along the composite [20,21].

Variation in mechanical properties between two parts molded with identical processing parameters, or between specimens obtained from different locations in the same part, may be attributed to porosity concentration in specific regions within the composite. Onedimensional numerical models have been used to predict the formation of voids resulting from flow disruption due to local permeability variations $[9,10]$. Many researchers have studied various fill parameters, the level of voids formed, and methods to minimize void content. The effects of mat architecture and liquid properties [11], vibrating mold walls, and mold surface treatments [22] have been presented. Ikegawa et al. proposed a technique to modify the RTM process, in which mold walls are compressed during filling, resulting in fewer voids [23].

Various studies have shown that pressure applied at different locations and times during filling significantly affects void formation. For example, vacuum assistance has been investigated as a method to decrease overall void content in molded parts. Chang and Hourng developed a model predicting that vacuum assistance would cause voids formed in the transverse impregnation of unidirectional fibers to shrink and disappear [6]; while Lundström and Gebart actually observed a decrease in measured void content in composites molded with vacuum assistance, with very few voids observed at the largest vacuum levels [7]. Similarly, the effects of injection pressure and injection rate or local velocity have been observed by both Patel and Lee [8], and Lee and Wei [3]. Patel and Lee used flow visualization to investigate flow patterns for several fiber mat architectures at different injection rates, while Lee and Wei investigated the effect of these variables on void formation in a radially expanding flow. However, the importance of post-fill cure pressure has not been studied in detail, even though cure pressure is known to have a significant effect on void content [7]. In the present study, packing pressure is found to have a more significant effect on the mechanical properties compared with the injection rate for the parts molded.

Glass preforms typically used in RTM contain fibers of $10-20 \mu \mathrm{m}$ in diameter, so voids with dimensions approximately $1-10 \mu \mathrm{m}$ are expected to form. Assuming a true randomness in the composite structure when selecting a particular cross-section, the void area fraction 
in the cross section is equivalent to the void volume fraction inside the part [24]. With this concept borrowed from stereology, Ghiorse [25] demonstrated that, dependent on the resolution of the system, image analysis is among the most accurate methods for assessing the true void content. Usually, the choice of the image resolution is a trade off between the demand for high resolution to detect voids of all sizes and the demand for coverage of a large area to minimize the number of micrographs necessary to cover the entire cross section [7].

In the current study, center-gated disk-shaped parts are fabricated at four different injection rates and four different packing pressures in order to determine the effects of these process parameters on the void content and mechanical properties in RTM. Mechanical testing data and characterization of voids with an optical microscope are used to determine the interrelation between void content and mechanical properties. This dependence could be utilized to develop predictive models for molding processes or used in controlling the molding to fabricate parts with better properties.

\section{EXPERIMENTAL SETUP}

\section{Molding Apparatus used to Fabricate RTM Parts}

An experimental molding setup is constructed to fabricate resin transfer molded disks. The setup, depicted in Figure 1, is composed of a molding press that injects a polymeric resin at either a specified flow rate or pressure, and a modular disk-shaped mold cavity. The molding press contains two hollow cylinders, which are for resin and curing agent, respectively. Plungers attached to the top plate of the press displace the resin and curing agent in the cylinders by equal distances when the top plate is moved. The flow from the two cylinders merges, then passes through a Statomix style motionless inline mixer

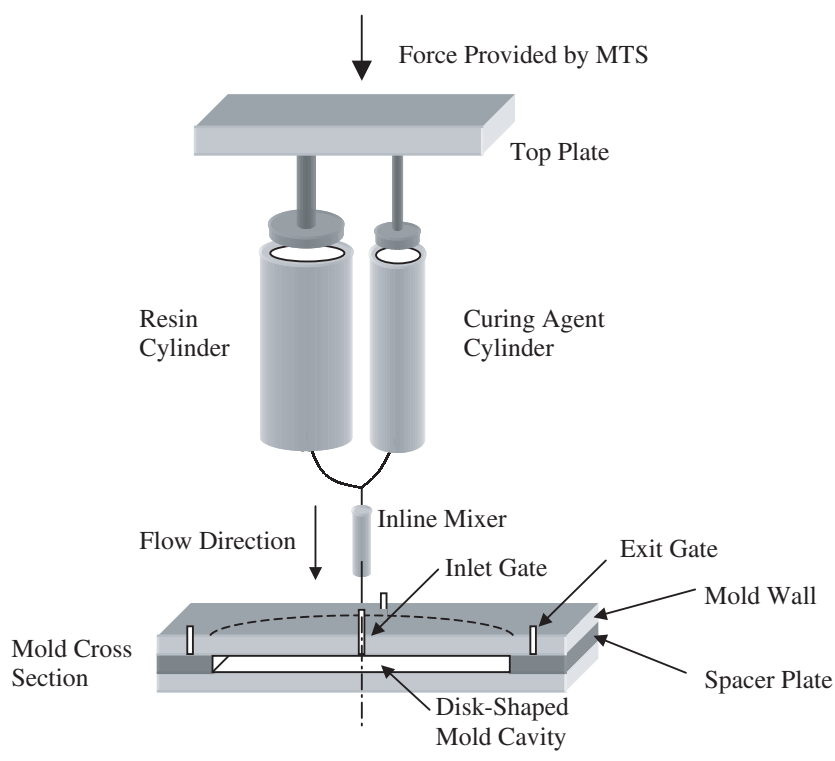

Figure 1. Experimental setup used to fabricate resin transfer molded disks. 
(Technical Resin Packaging, Inc.) which facilitates thorough mixing of the two components. The mixture is then injected into the center of the disk-shaped cavity. The entire press is mounted on a materials testing system (MTS) machine, which provides the force necessary to inject the mixed resin. The MTS machine is computer controlled, and is run with either constant linear velocity, resulting in constant volume flow rate, or at constant force for a fixed inlet pressure.

The mold cavity, depicted in Figure 2, is constructed by placing an aluminum spacer plate having a thickness of $3.18 \mathrm{~mm}(0.125 \mathrm{in})$ between two $6.35 \mathrm{~mm}(0.25 \mathrm{in})$ thick aluminum mold walls. A $15.24 \mathrm{~cm}$ (6.0 in) diameter circle is cut from the center of the spacer plate to form the disk-shaped cavity. After filling, holding a packing pressure on the mold has been demonstrated to produce higher quality parts [26]. In order to study this effect, an O-ring groove is machined into each mold wall to form a pressure tight seal. An inlet gate is drilled and tapped for a hose fitting in the top mold wall at the center of the disk-shaped cavity. Four vents are also drilled in the top mold wall and tapped for hose fittings. Vents are positioned evenly around the cavity at a radius of $8.89 \mathrm{~cm}(3.5 \mathrm{in})$. Chopped-strand glass mats $\left(1.5 \mathrm{oz} / \mathrm{ft}^{2}\right.$, Fiberglast part \#250) cut into $15.24 \mathrm{~cm}$ (6.0 in) diameter circles are layered in the mold cavity prior to filling, to reinforce the resin transfer molded parts. Mats have a randomly distributed, in-plane fiber orientation. For this study, four mat layers are stacked in the cavity, representing a $21 \%$ fiber volume fraction.

An epoxy resin, EPON 815C, manufactured by Shell Chemicals, is selected for the molding experiments. Attractive properties of this resin include its low viscosity, which facilitates moderate injection pressures, and its low toxicity. The desired gel time of about $20 \mathrm{~min}$ is achieved by selecting EPICURE 3282 (Shell Chemicals) as the curing agent. The internal diameters of the two stainless steel cylinders are machined to 55.47 and $25.53 \mathrm{~mm}$ in order to achieve the appropriate mix ratio, 4.7 to 1 by volume, of resin to curing agent. Cylinders contain enough resin and curing agent to mold six of the disks during a single stroke. Thus, six disks are prepared in each run using six identical molds.

The parts are molded at constant flow rates, with typical fill times ranging between 20 and $900 \mathrm{~s}$. After filling, the mold is left attached to the press, the vents closed, and the selected packing pressure applied for $300 \mathrm{~s}$. The inlet hose is then clamped to maintain the pressure, and the part is left in the mold for $24 \mathrm{~h}$ before demolding. The part is then cured for a minimum of $24 \mathrm{~h}$ more before the specimens are machined and polished, and for at least one week before testing to ensure complete post curing. The entire process is performed at room temperature. A representative figure depicting the increase in molding force for a $300 \mathrm{~s}$ mold filling is shown in Figure 3. A representative inlet pressure is obtained by dividing the molding force by the cross sectional area of the two plungers $\left(29.3 \mathrm{~cm}^{2}\right)$. After an initial rise, the injection force increases gradually for the first $300 \mathrm{~s}$

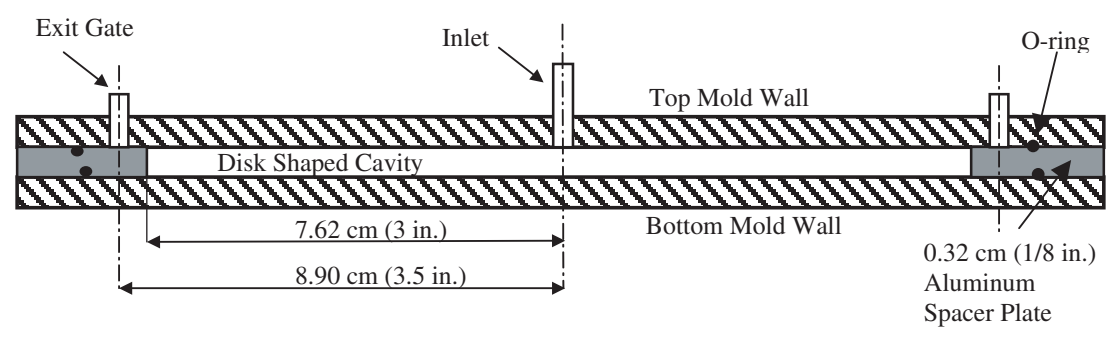

Figure 2. Cross section of the disk-shaped mold cavity used to fabricate resin transfer molded disks. 


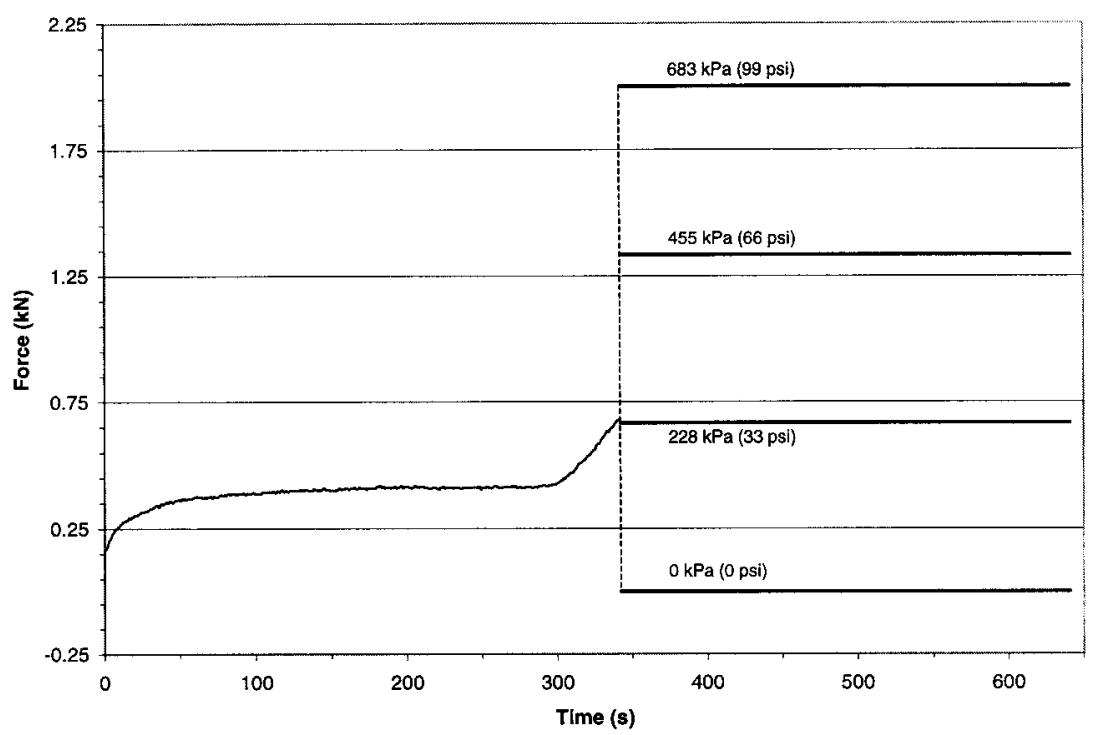

Figure 3. Representative injection force during mold filling showing four different packing pressures after filling is completed.

during the mold filling at constant flow rate. Between 300 and $350 \mathrm{~s}$, the force increases more substantially, as the mold is nearly full, and resin is forced, still at constant injection rate, between the spacer plate and mold wall into all exit vents. After the mold has completely filled, one of four packing pressures (shown in Figure 3) is selected, and the corresponding constant force is applied for $300 \mathrm{~s}$.

\section{Mechanical Testing of Molded Parts}

Each molded disk is cut into five $11.43 \times 1.27 \times 0.318 \mathrm{~cm}(4.50 \times 0.50 \times 0.125 \mathrm{in})$ rectangular tensile test specimens according to ASTM D3039/D3039M standards [27]. Figure 4 depicts the relative spatial positions of the five specimens within the molded disk. Angular position of the specimen array within each disk is arbitrary due to random planar fiber orientation. Each disk yields one specimen in the center of the disk, with the inlet passing through the center of the specimen (at position 3). Two position 2 specimens are arranged symmetrically on either side of the center specimen, and two position 1 specimens are located near the edge of the disk. Specimens are cut from the disks using a vertical milling machine to yield the best tolerances possible. Subsequently, each specimen is polished, and five thickness and width measurements are taken at different locations on the specimen using a micrometer. Typical specimen thicknesses vary between 0.3 and $0.4 \mathrm{~cm}$ due to slight mold bulging, resulting from variations in the injection pressure and the difference in the compression on the O-rings. The average thickness is $0.34 \mathrm{~cm}$, with a standard deviation of 0.013 .

Position 1 and 2 specimens are tested under tension using the MTS machine. Position 3 is less suited to tensile testing, due to possible defects at the inlet gate, where the cured resin column contained in the inlet must be broken off during demolding. Positions 1 and 2 represent directions more or less orthogonal to the flow. The force is ramped linearly 


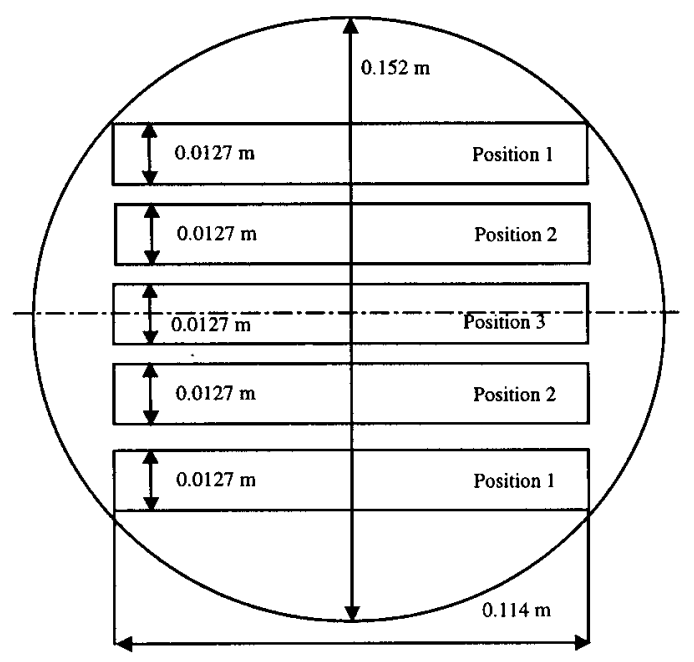

Figure 4. Diagram of a molded disk depicting locations of the tensile test specimens.

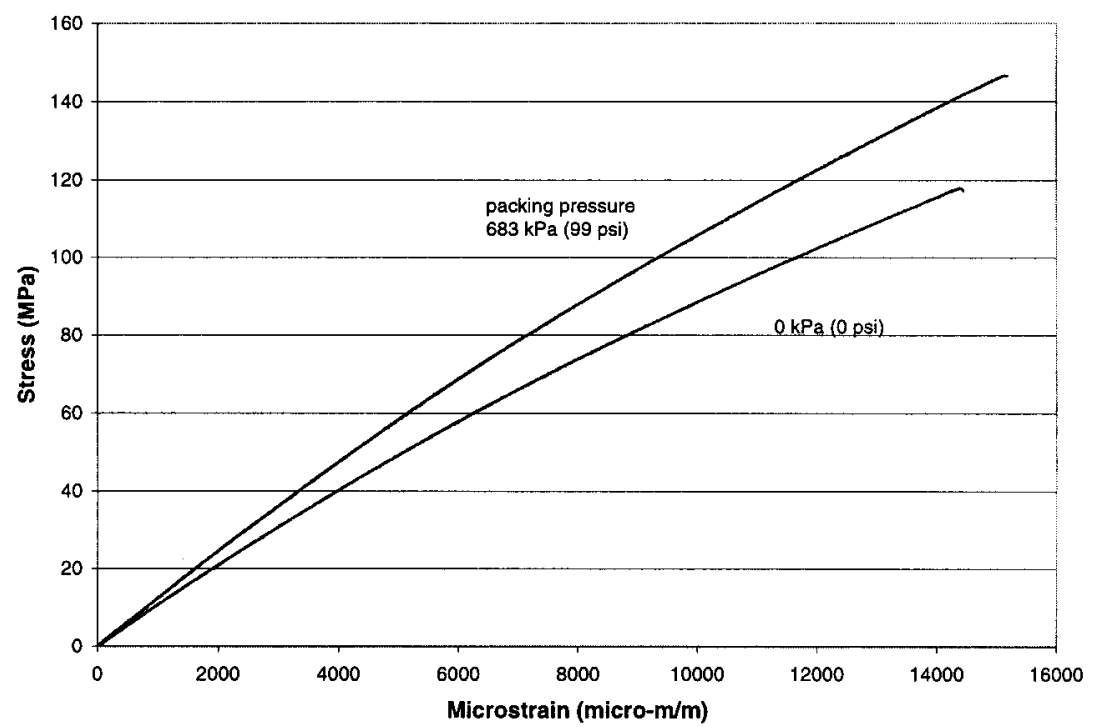

Figure 5. Representative stress-strain curves of two position 1 specimens molded at two different packing pressures.

from 0 to $8.90 \mathrm{kN}(0-2000 \mathrm{lbs})$ over $120 \mathrm{~s}$. Typically maximum loads for specimens used in this study are around $4.5 \mathrm{kN}(1000 \mathrm{lbs})$. An extensometer is attached over a $2.54 \mathrm{~cm}$ (1.00 in) span at the center of the specimen in order to measure the strain directly. The stress is calculated by dividing the force by the cross sectional area, and stress-strain curves are generated for each specimen. Two representative stress-strain curves for position 1 specimens molded using different packing pressures are depicted in Figure 5. From the data, maximum stress is obtained, and elastic modulus is calculated over the linear region between 1000 and 3000 microstrain, as specified by ASTM D3039/D3039M. 


\section{Microscopic Analysis to Quantify Void Content}

Microscopic analysis performed on specimens cut from position 3 in each kind of molded part is used to assess the void volume fraction, as well as other important parameters such as void size, shape, and roundness. Voids with different characteristics are likely to originate from different sources during filling, and will contribute to mechanical properties and failure mechanisms differently. For example void size directly affects void mobility and thus, void distribution. Void shape affects mechanical properties, with irregular voids being more likely to induce early crack formation. Cross sections of each specimen are embedded using a commercial resin (Embed 812-DER 736), and then machined to have a flat base surface for further polishing. A series of silicon carbide pastes of different grit sizes ranging from 240 to 1200 insured a smooth finish. Finish with aluminum powder was not necessary to obtain good surfaces for the composite used in this study. Once polished, the cross sections are analyzed and pictures of individual voids taken at 100x with an optical microscope (Nikon OPTIPHOT-2) and attached CCD camera (Hammatsu C2400). Individually captured pictures are processed with the aid of an image analysis software (Optimas v4.1), which has features such as area morphometry that enable not only void area measurement, but also the classification of the voids according to their size and shape (i.e., circularity, $j=$ perimeter $^{2} /$ area). Assuming a linear equivalence, the volumetric void content was simply taken as the percentage ratio of the total cross sectional void area divided by the cross sectional area of the entire sample.

Voids are classified into three categories based on their circularity values: circular, ellipsoidal/irregular, and very irregular. A circularity of 12.58 is used for circular voids, while values greater than 20 were assigned to highly irregular voids. Intermediate values represent ellipsoidal to irregular geometries. The longest axis is used as the sole indicator to account for void size. Representative images obtained from the microscopic analysis are depicted in Figure 6. Figure 6(a) depicts the matrix containing fibers perpendicular to the cross section, seen as small circular dots in the figure. Elliptical white objects observed in the figure are fibers oriented nearly parallel to the cross section. Fibers with similar orientations are observed in close proximity to one another in the image. These are fiber tows containing groups of similarly-aligned fibers bundled together within the random mats.

A large circular void, with a diameter of approximately $100 \mu \mathrm{m}$, about 10 times the fiber diameter, and a smaller irregular void are visible in Figure 6(b). These voids are representative of those found at 0 packing pressure. At slightly higher packing pressure, $228 \mathrm{kPa}$, fewer large, circular voids are found. Most voids are smaller and slightly irregular, such as the one depicted in Figure 6(c). At higher packing pressures, voids are irregular and trapped in fiber tows (Figure 6d).

\section{RESULTS AND DISCUSSION}

\section{Effect of Injection Rate on Mechanical Properties}

Initially, the disks are molded at different flow rates in order to ascertain the effect of this parameter on mechanical properties. Four different constant injection rates of 0.067 , $0.2,0.6$, and $1.0 \mathrm{~cm}^{3} / \mathrm{s}$, representing more than an order of magnitude change in this parameter, are used. These injection rates result in fill times of approximately 900, 300, 100 
(a)

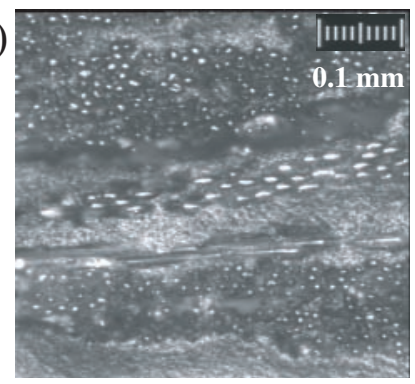

(b)

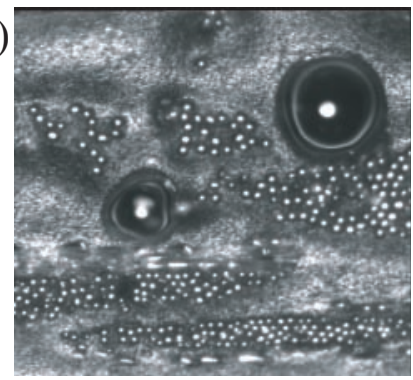

(c)

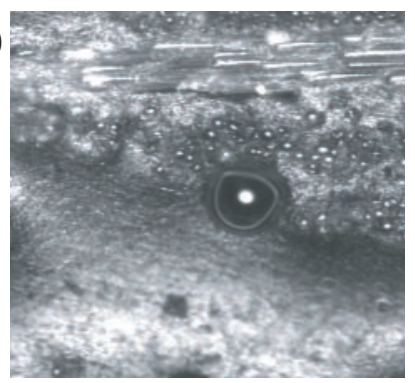

(d)

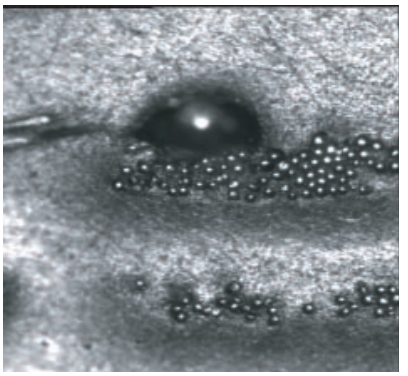

Figure 6. Representative microscopic images obtained from specimen cross-sections at 100x magnifications. (a) Example of fibers perpendicular to and parallel to cross section; (b) Large, circular void at $0 \mathrm{kPa}$ packing pressure; (c) Smaller, circular void at $228 \mathrm{kPa}$; (d) Irregular in-tow void at $683 \mathrm{kPa}$.

\section{Table 1. Molding parameters used to determine effect of volume flow rate on mechanical properties.}

\begin{tabular}{|c|c|c|}
\hline $\begin{array}{l}\text { Flow Rate } \\
\left(\mathrm{cm}^{3} / \mathrm{s}\right)\end{array}$ & $\begin{array}{l}\text { Fill Time } \\
\text { (s) }\end{array}$ & $\begin{array}{l}\text { Packing Pressure } \\
(\mathrm{kPa})\end{array}$ \\
\hline 0.067 & 900 & 228 \\
\hline 0.2 & 300 & 228 \\
\hline 0.6 & 100 & 228 \\
\hline 1.0 & 60 & 228 \\
\hline
\end{tabular}

and $60 \mathrm{~s}$ respectively. The 100 and $60 \mathrm{~s}$ fill times are consistent with fill times observed in industrial molding of small to medium sized RTM parts. Industrial molding is frequently performed at constant injection pressure, in which case the non-uniform injection rate can be calculated from the analytical solution given by Adams et al. [28]. The slower fill times are expected to yield better overall part quality as fewer voids will be entrapped by a slower moving fluid front. Disks in this series are molded with a packing pressure of $228 \mathrm{kPa}$ (33 psi). All pressures given in this study are gage pressures. Molding parameters for this series are tabulated in Table 1. At least five disks are molded at each flow rate, and the measured ultimate tensile strength and elastic modulus at each velocity are averaged from all specimens to reduce statistical error. Positions 1 and 2 have two symmetric specimens from each disk, resulting in a total of 10 specimens to average for each data point. However, data from specimens that broke at or near the MTS grips are discarded.

Tensile strength based on volume flow rate for positions 1 and 2 is depicted in Figure 7. Error bars are calculated using a $95 \%$ confidence interval, and a maximum error of about 


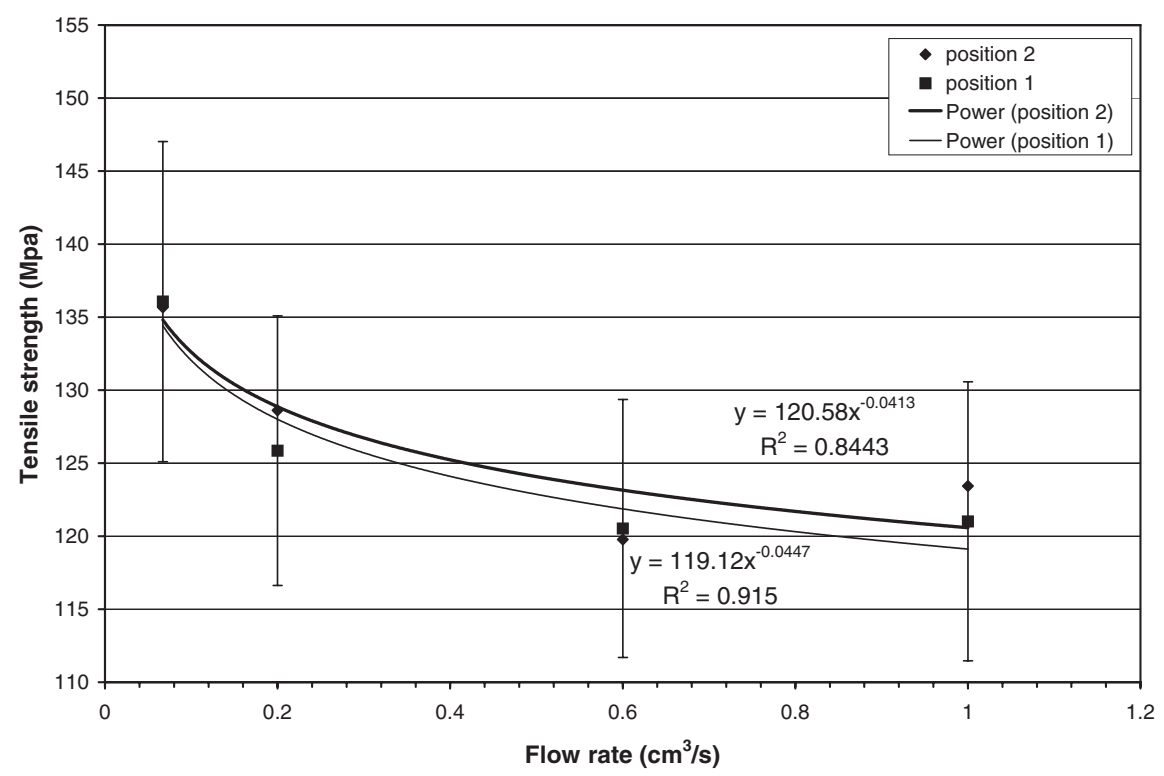

Figure 7. Ultimate tensile strength variation with injection flow rate for positions 1 and 2. Packing pressure $=228 \mathrm{kPa}$.

$9 \%$ is observed. Tensile strength results from positions 1 and 2 are similar, and exhibit an exponential decrease in strength with increased flow rate. The decrease in strength is about $13 \%$ from 134 to $119 \mathrm{MPa}$ for position 1, over the range of flow rates studied. A power law curve is seen to fit the data well, with all experimental points fitting the curve within the associated uncertainties. The decrease in strength at higher flow rates is most likely due to increased void formation resulting from more air being trapped by the faster moving fluid front. The slight increase in strength at the highest flow rate may be due to statistical variation in the data resulting mainly from inhomogeneities in local mat architecture. Gauvin et al. report that surface densities of many commercially available random mats can vary locally by a factor of as much as $100 \%$ [29]. This difference leads to variation in volume fraction and permeability throughout the part. Mats are weighed to ensure the correct overall fiber content, care is taken to select similar mats, and multiple tests are conducted to help reduce statistical uncertainty. However, the variation in local mat architecture seems to affect the statistical variation of the data. Another potential explanation of the increase in strength at flow rate of $1 \mathrm{~cm}^{3} / \mathrm{s}$ is that shear thinning resins may penetrate into fiber bundles more easily, thus reducing void formation [3].

Due to very similar tensile results observed between positions 1 and 2, data from these two positions are combined to reduce uncertainty and generate the single curve depicted in Figure 8. Combining data from the two positions results in a reduction in the average observed uncertainty for a $95 \%$ confidence interval to $5.5 \%$. It has been shown that local velocity of the fluid front, as it passes a particular location, is one of the primary factors influencing lag-lead or fingering [30], and thus void formation and properties in the cured part. Change in local velocity between positions 1 and 2 is small compared with the change in velocity between disks molded at different injection rates, thus void fraction and mechanical properties from positions 1 and 2 are expected to be similar. It may be considered that fibers in the mats may translate or rotate due to the flow field during 


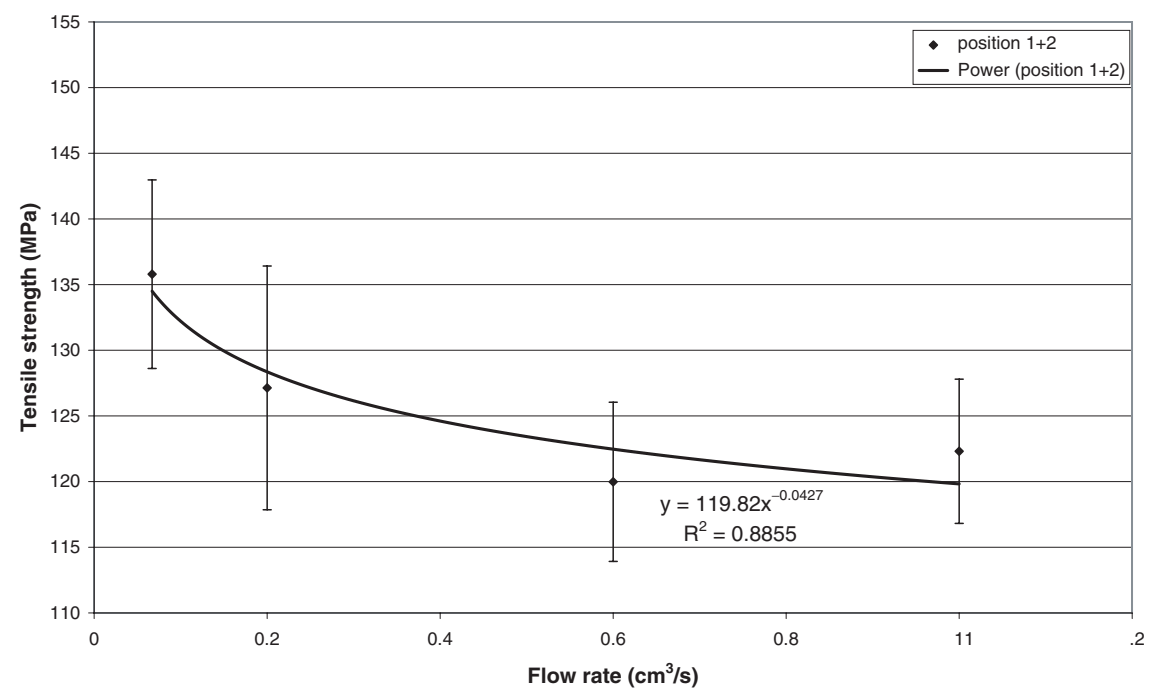

Figure 8. Ultimate tensile strength variation with injection flow rate averaged over positions 1 and 2. Packing pressure $=228 \mathrm{kPa}$.

molding, causing fiber concentration or orientation differences throughout the part. However, similarities between properties at these two locations and microscopic observation of the samples indicate that fiber mobility and rotation is not a significant concern over the scale and parameters considered in the present study.

Stiffness data from these specimens dependent on injection rate is depicted in Figure 9. Similar to strength behavior, a power law fit based on injection rate is found to represent the data well. Again, error bars are drawn with a $95 \%$ confidence interval, and data from positions 1 and 2 are averaged due to their similar behavior, thus reducing uncertainty. The average statistical error is about $3.6 \%$, which is lower compared with the uncertainty in strength results. The stiffness is also found to decrease with increased velocity. A slightly greater reduction with increased flow rate is observed compared with the decrease in strength data. Stiffness drops about $14 \%$ from 11.5 to $10.1 \mathrm{GPa}$ over the range of injection rates used.

\section{Effect of Packing Pressure on Mechanical Properties and Void Content}

Twelve additional disks are molded at an injection rate of $0.2 \mathrm{~cm}^{3} / \mathrm{s}$ with varied packing pressures of 0,455 , and $683 \mathrm{kPa}(0,66$, and $99 \mathrm{psi})$ to determine the effect of packing pressure on properties of the final part. The data presented in the previous section, obtained from disks molded at a flow rate of $0.2 \mathrm{~cm}^{3} / \mathrm{s}$ and a packing pressure of $228 \mathrm{kPa}$ (33 psi), provides an additional packing pressure for comparison. Table 2 contains the molding parameters for these four sets of disks. Position 1 and 2 specimens from these disks are tested under tension to determine the mechanical properties, while position 3 specimens are analyzed optically for void characterization.

Packing pressure is found to have a greater effect on the mechanical properties compared with injection rate. The ultimate tensile strength based on packing pressure for the average of positions 1 and 2 is depicted in Figure 10. Error bars in Figure 10 are 
Kristian A. Olivero et al.

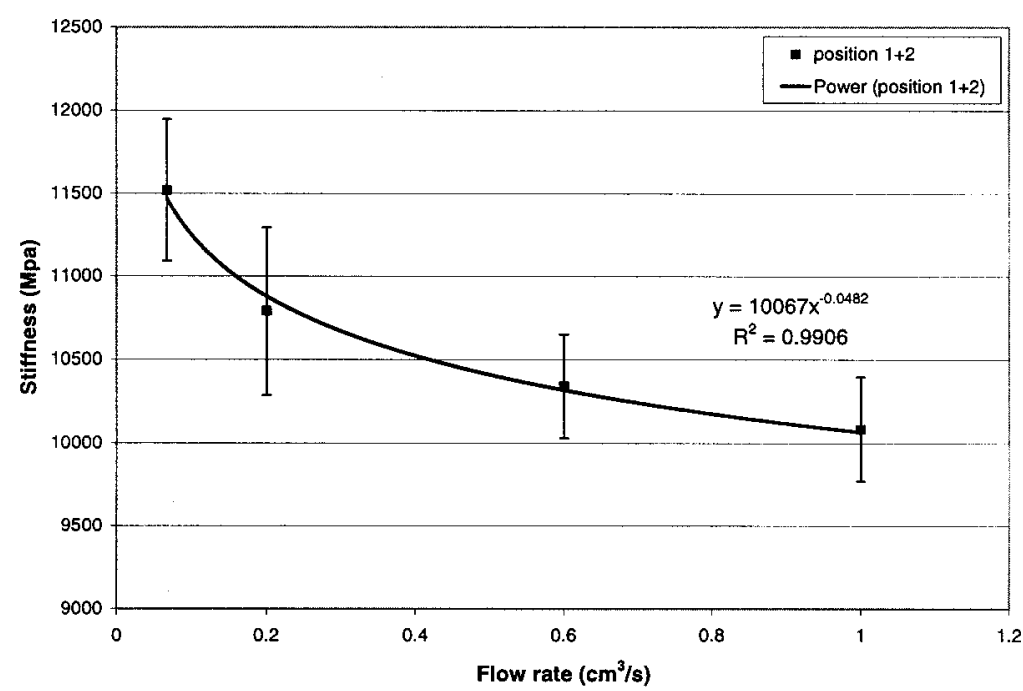

Figure 9. Stiffness variation with injection rate averaged over positions 1 and 2. Packing pressure $=228 \mathrm{kPa}$.

Table 2. Molding parameters used to determine effect of packing pressure on mechanical properties and void content.

\begin{tabular}{lcc}
$\begin{array}{l}\text { Flow Rate } \\
\left(\mathbf{c m}^{\mathbf{3}} \mathbf{s}\right)\end{array}$ & $\begin{array}{c}\text { Fill Time } \\
\mathbf{( s )}\end{array}$ & $\begin{array}{c}\text { Packing Pressure } \\
\mathbf{( k P a )}\end{array}$ \\
\hline 0.2 & 300 & 0 \\
0.2 & 300 & 228 \\
0.2 & 300 & 455 \\
0.2 & 300 & 683 \\
\hline
\end{tabular}

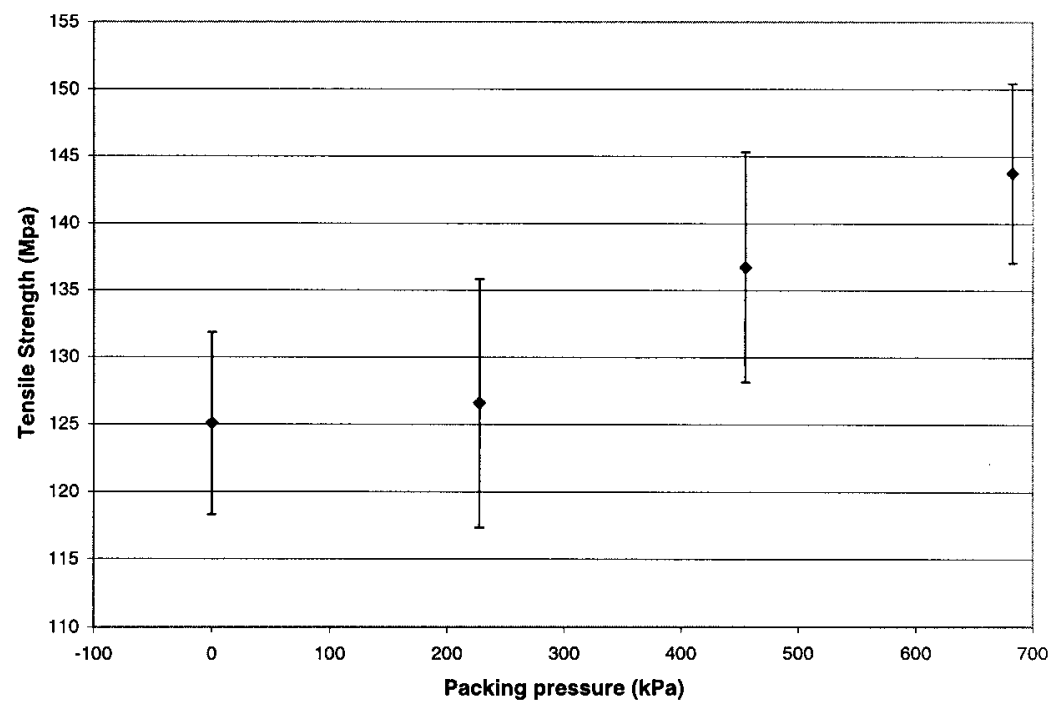

Figure 10. Ultimate tensile strength variation with packing pressure averaged over positions 1 and 2. Flow rate $=0.2 \mathrm{~cm}^{3} / \mathrm{s}$. 
calculated with a $95 \%$ confidence interval. Tensile strength increases by a total of $15 \%$ from $125 \mathrm{MPa}$ at $0 \mathrm{kPa}$ packing pressure, to $144 \mathrm{MPa}$ at $683 \mathrm{kPa}$ packing pressure. The increase in strength between 0 and $228 \mathrm{kPa}$ pressure is observed to be much smaller (i.e., $1.3 \%$ ) than the increase in strength between 228 and $455 \mathrm{kPa}$ (i.e., $8.0 \%$ ), and between 455 and $683 \mathrm{kPa}$ (i.e., $5.1 \%$ ). This indicates a minimum packing pressure may be required to observe improvements in mechanical properties. There is a significant improvement in strength between 455 and $683 \mathrm{kPa}$, the highest pressure used; thus even higher strengths may be obtained at packing pressures higher than $683 \mathrm{kPa}$. It is believed that packing pressure improves strength mainly by reducing the size and quantity of voids. Increased pressure may also improve wetting of fibers by forcing resin into fiber bundles that are not fully wetted during filling. An upper limit on useful packing pressures is anticipated, due to limits on void reduction and complete wetting.

Figure 11 depicts elastic modulus based on packing pressure for the average of position 1 and 2 data. Similar to the results for disks molded at different injection rates, the average observed error for stiffness is lower compared with strength results. An average statistical error of about $4 \%$ is obtained, with little variation in error at different packing pressures. At the maximum packing pressure, a $13 \%$ increase in stiffness to $12.2 \mathrm{MPa}$ is observed which is slightly less than the percentage increase in strength. There is a small increase in stiffness between 0 and $228 \mathrm{kPa}$ packing pressure, further indicating a minimum packing pressure is required for the benefits to be observed. There is also a small increase of $2.5 \%$ in stiffness over the final interval from 455 to $683 \mathrm{kPa}$, indicating a possible upper limit on improved stiffness is being approached at this level of packing.

\section{Correlation Between Void Content and Mechanical Properties}

Microscopic analysis is performed on specimen cross sections from each of the four packing pressures. Void content is found to decrease with increased pressure, with fairly

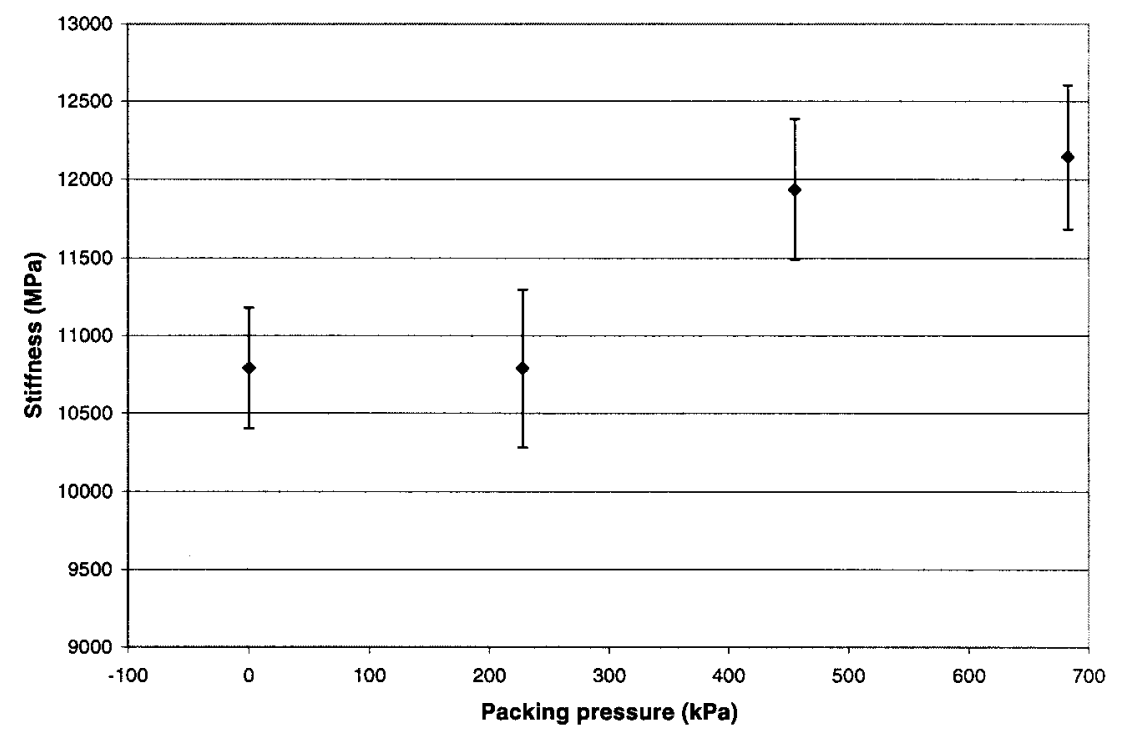

Figure 11. Stiffness variation with packing pressure averaged over positions 1 and 2 . Flow rate $=0.2 \mathrm{~cm}^{3} / \mathrm{s}$. 
low void fractions of $0.72,0.52,0.32$, and $0.34 \%$ measured for packing pressures of 0,228 , 455 , and $683 \mathrm{kPa}$, respectively. Figure 12 depicts an exponentially decaying function of void fraction based on the four post-fill cure pressures used in the current study. Error bars are not drawn in Figure 12, as each measurement is obtained from a single disk molded at the corresponding packing pressure. The three-parameter exponential curve is fit to the data by minimizing the sum of the squared differences between the measured and analytical values using the solver feature in Microsoft Excel. It is clear from this graph that the application of higher resin pressures after the molds have been filled further reduces the already low porosity inside the composite parts. This particular reduction in porosity can be correlated with the increase in the post-fill cure pressure by considering the establishment of a high local pressure that shrinks individual bubbles and possibly cause smaller voids to dissolve into the resin. Although the radial distribution of voids inside the parts was not measured in this study, a careful examination of the size and shape distribution of the voids encountered in each one of the specimens would give some indications of the actual mobility of the voids. As can be seen in Figure 13, the combined relative percentage of both circular and ellipsoidal bubbles decreases as the post-fill pressure increases. Both circular and ellipsoidal voids are usually located in the inter-tow spaces. Thus, a smaller relative presence of these type of voids at high post-fill pressures is indicative of more voids in the interstitial areas. It is observed that the contribution of very irregular voids is increased at higher packing pressures, although the total void fraction and number of voids has decreased. It seems that the irregular voids, which are typically found inside fiber bundles, are less susceptible to removal by increased pressure compared with the more circular voids found in the interstitial areas.

A second characterization of void content with increased post-fill cure pressure is presented in Figure 14. Void shape distribution is tabulated at each of the packing pressures. Circular and ellipsoidal voids have sizes that are mostly in the range 50-150 $\mu \mathrm{m}$. Based on the total number of voids in this particular range, it is clear that as long as the local pressure is increased, the contribution of the small-to-medium circular voids decreases,

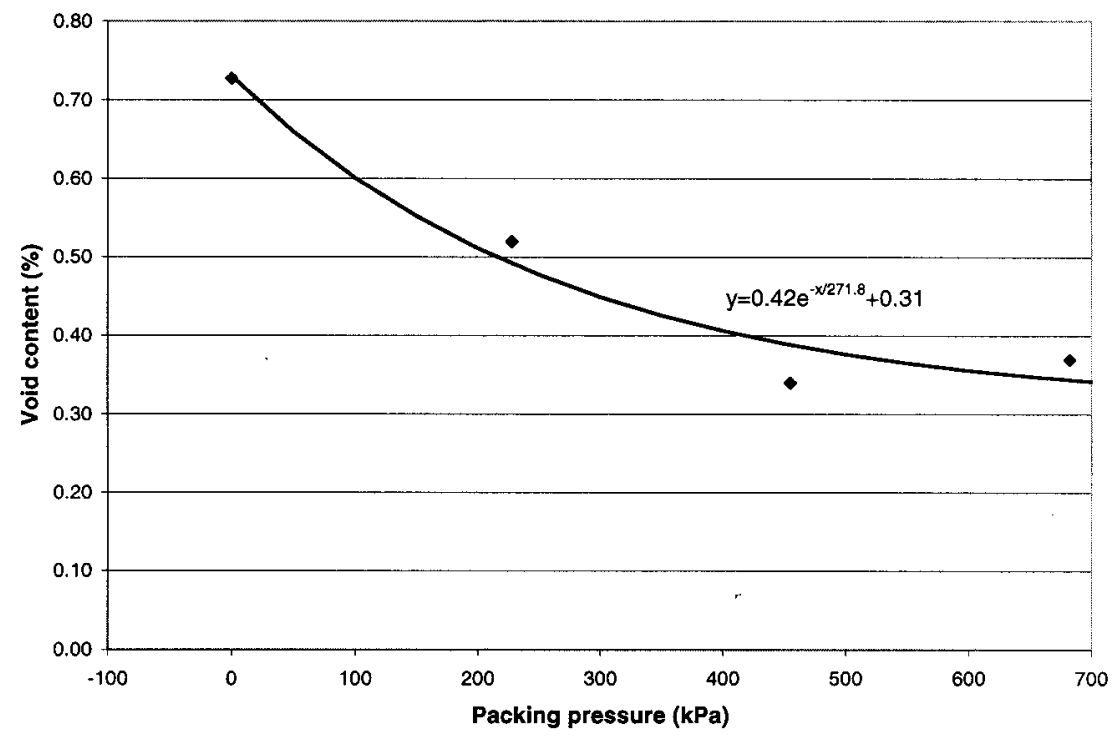

Figure 12. Void area fraction variation with packing pressure. Flow rate $=0.2 \mathrm{~cm}^{3} / \mathrm{s}$. 


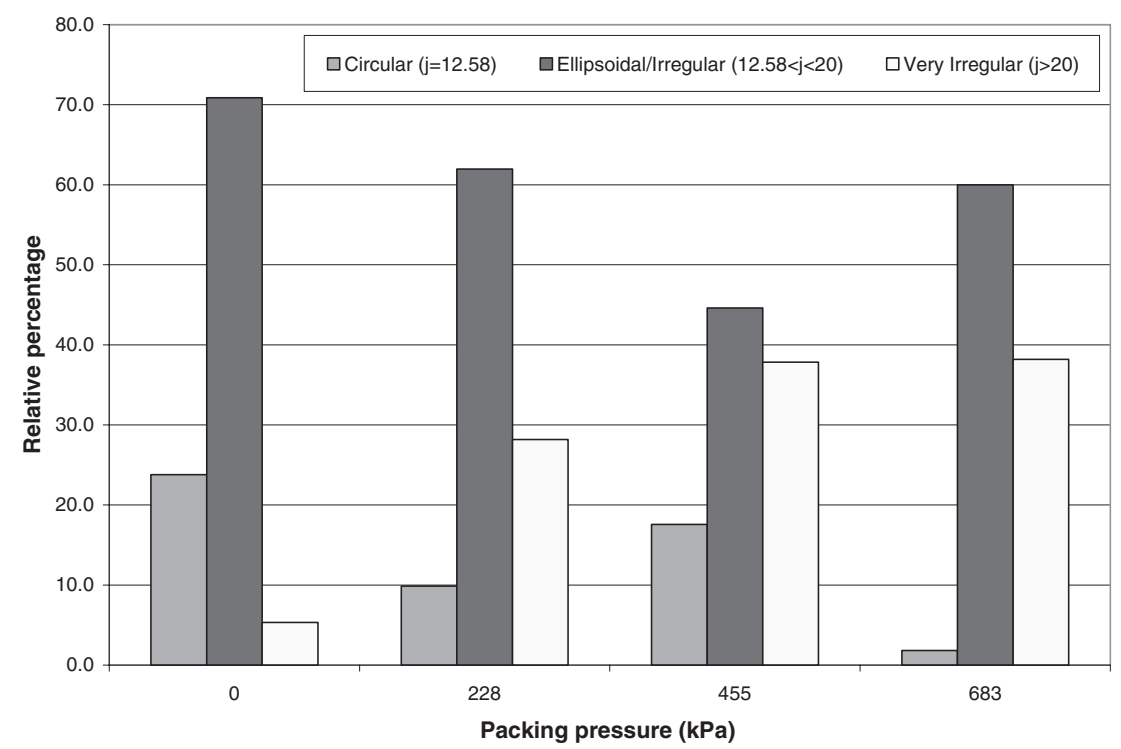

Figure 13. Void shape distribution based on packing pressure. Flow rate $=0.2 \mathrm{~cm}^{3} / \mathrm{s}$.

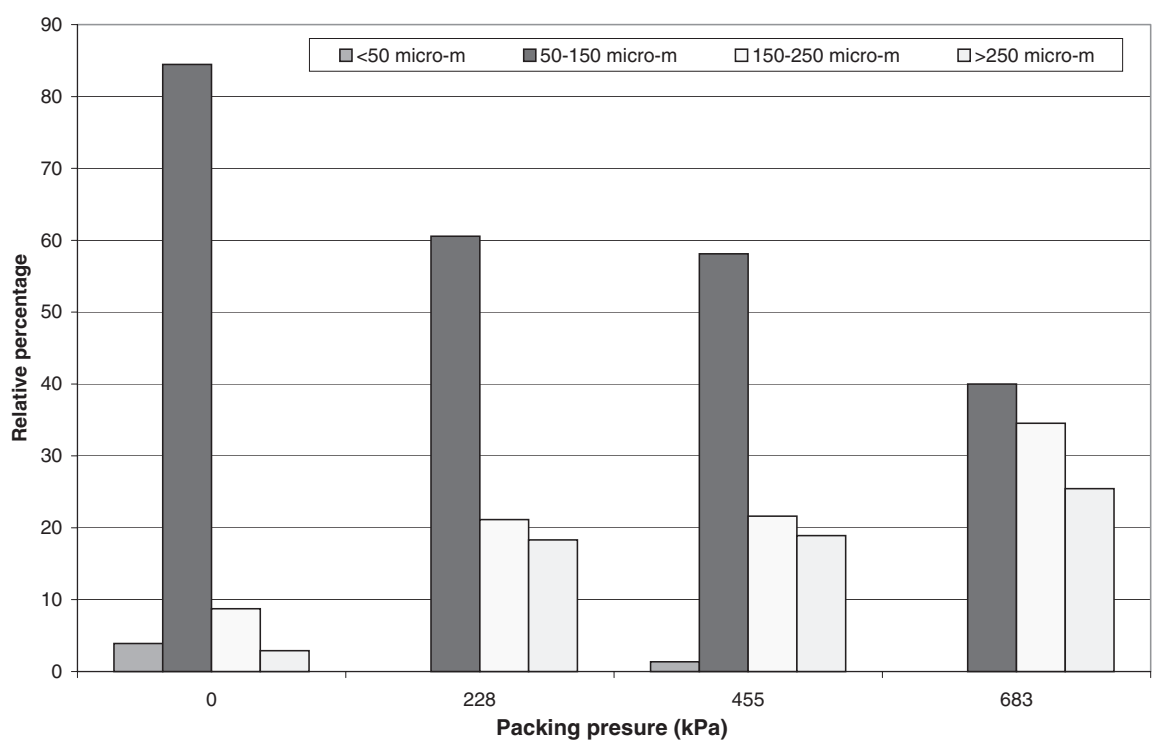

Figure 14. Void size distribution based on packing pressure. Flow rate $=0.2 \mathrm{~cm}^{3} / \mathrm{s}$.

thus increasing the relative percentage of large irregular voids. During image analysis the large irregular voids are observed primarily in the intra-tow spacing. Varna et al. [20] recently suggested that laminates with highest average content of voids also had the highest content of spherical voids, which concentrated in regions away from the inlet gate. Our results, presented in Figures 13 and 14, agree with those presented by Varna et al. These data suggest increasing the post-fill cure pressure is a potentially viable method for removing or compressing entrapped air, thus decreasing void content of a molded part. 
As a consequence of decreased void content at higher packing pressures, it is expected that the mechanical properties would be improved. The strength and stiffness are directly correlated with the measured total void content to investigate this effect. In Figure 15, the strength is observed to decrease exponentially by nearly $15 \%$ from 143 to $125 \mathrm{MPa}$ with increased void content. The parameters from the exponential curve fits in both Figures 15 and 16 are found by minimizing the sum of the squared differences between the measured and analytical values using the solver feature in Microsoft Excel. The maximum strength observed at the minimum void content, $0.35 \%$, represents the highest packing pressure.

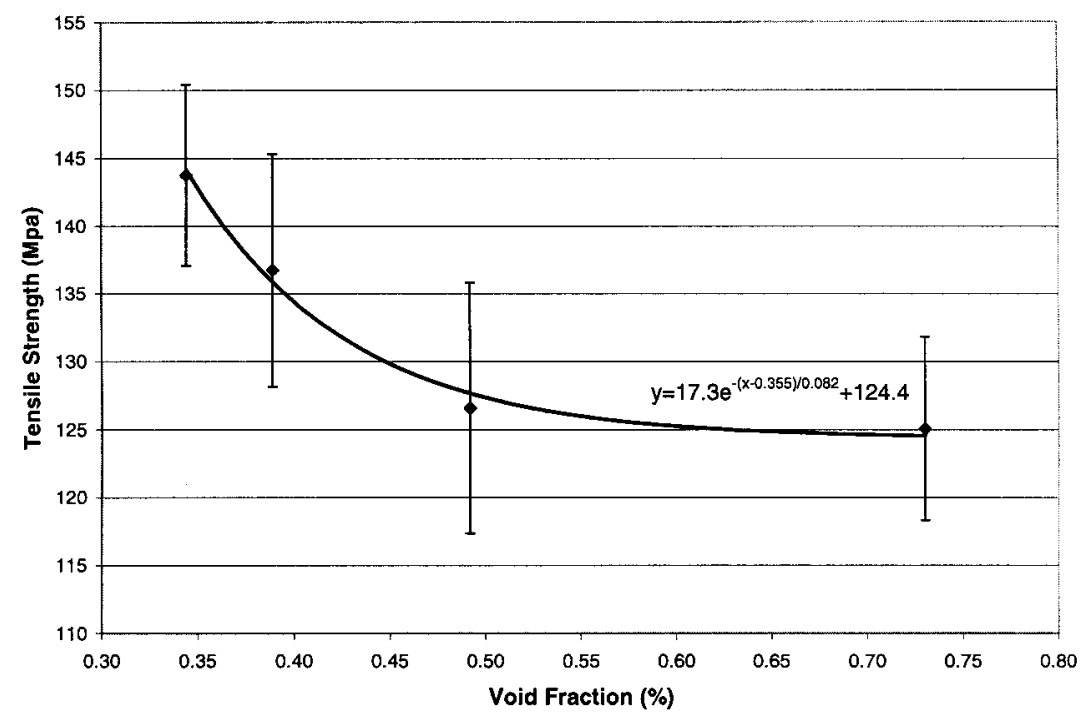

Figure 15. Ultimate tensile strength variation with void fraction averaged over positions 1 and 2. Flow rate $=0.2 \mathrm{~cm}^{3} / \mathrm{s}$.

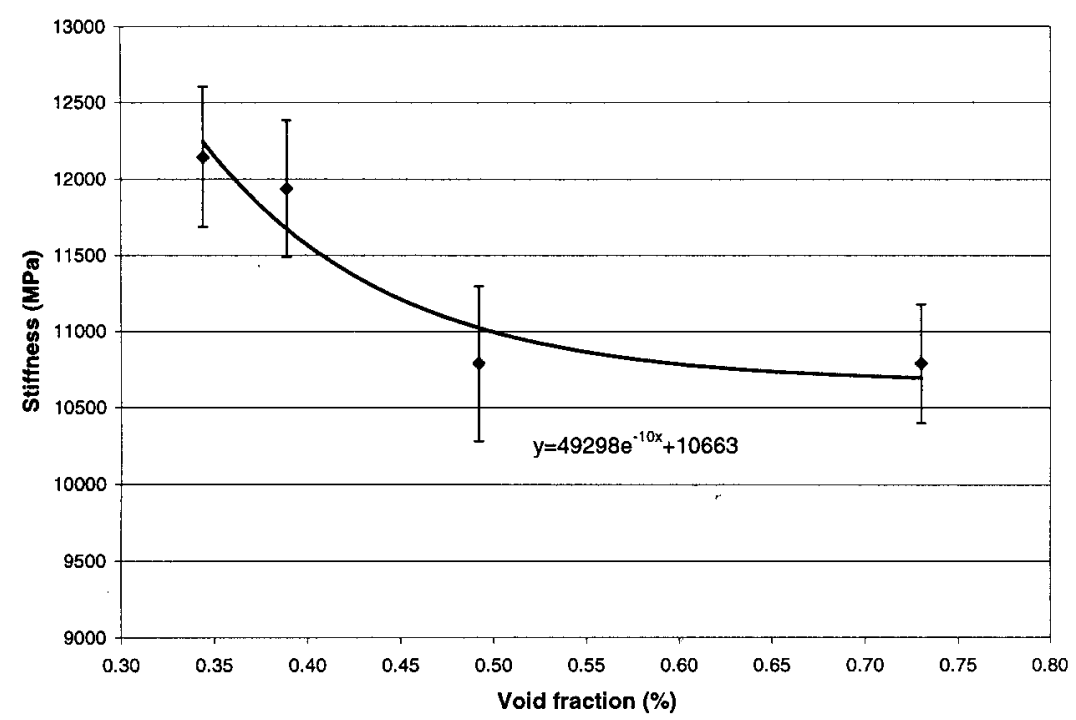

Figure 16. Stiffness variation with void fraction averaged over positions 1 and 2 . Flow rate $=0.2 \mathrm{~cm}^{3} / \mathrm{s}$. 
The stiffness based on void content, depicted in Figure 16, demonstrates a similar behavior. An exponential decrease in modulus from about 12.2 to $10.7 \mathrm{GPa}$ is observed as the void content doubles from about 0.35 to $0.73 \%$. These results clearly show that the decreased void content obtained at higher post-fill cure pressures directly translate to improved mechanical properties of the final part.

\section{CONCLUDING REMARKS}

Increased injection rate is found to have a detrimental effect on the mechanical properties of resin transfer molded composite disks. Both the stiffness and tensile strength are measured to decrease by 14 and $13 \%$, respectively, with 15 -fold increase in injection rate. At higher injection rates, the fluid front moves faster and traps more air leading to increased void formation. Increased packing pressure is presented as a method for decreasing void content in RTM process. Increased packing pressure is observed to improve mechanical properties, with $15 \%$ increase in strength and $13 \%$ increase in elastic modulus over the pressure ranges studied. This is due to lower void content which decreased from 0.72 to $0.35 \%$ by increasing pressure from 0 to $683 \mathrm{kPa}$. It is observed that there are fewer, more irregularly shaped voids at higher packing pressures. Average void size is also observed to increase at higher packing pressures. Close examination of the data and micrographs reveals that this is due to a reduction in total number of small voids, while the quantity of larger irregular voids remains unchanged. Strength and stiffness are correlated directly with void fraction. An exponential decrease in both strength and stiffness is observed as the void fraction increased from 0.35 to $0.72 \%$.

\section{ACKNOWLEDGEMENTS}

This work has been partially supported by the NASA Microgravity Materials Research Program under Grant NAG8-1271.

\section{REFERENCES}

1. Calhoun, D.R., Yalvaç, S., Wetters, D.G. and Raeck, C.A. (1996). Polymer Composites, 17(1): $11-22$.

2. Loos, A.C. and Springer, G.S. (1983). Journal of Composite Materials, 17: 135-169.

3. Lee, C.-L. and Wei, K.-H. (2000). Polymer Engineering and Science, 40(4): 935-943.

4. O’Donnell, H.J. and Baird, D.G. (1996). Polymer Engineering and Science, 36(7): 963-978.

5. Patel, N., Rohatgi, V. and Lee, L.J. (1993). Polymer Composites, 14(2): 161-172.

6. Chang, C.-Y. and Hourng, L.-W. (1998). Polymer Engineering and Science, 38(5): 809-818.

7. Lundström, T.S. and Gebart, B.R. (1994). Polymer Composites, 15(1): 25-33.

8. Patel, N. and Lee, L.J. (1996). Polymer Composites, 17(1): 104-114.

9. Parnas, R. and Phelan, F. (1991). SAMPE Quarterly, 22(2): 53-60.

10. Moon, K., Lee, W. and Hanh, T. (2000). Composites Science and Technology, 60: 2427-2434.

11. Patel, N. and Lee, L.J. (1995). Polymer Composites, 16(5): 386-399.

12. Callus, P.J., Mouritz, A.P., Bannister, M.K. and Leong, K.H. (1999). Composites Part A, 30: 1277-1287. 
13. De Oliveira Simões, J.A. and Marques, A.T. (2001). Composites Part A, 32: 655-662.

14. Hillermeier, R.W. and Seferis, J.C. (2001). Composites Part A, 32: 721-729.

15. Margueres, Ph., Meraghni, F. and Benzeggagh, M.L. (2000). Composites Part A, 31: 151-163.

16. Thomason, J.L. (1995). Composites, 26: 467-475.

17. Ghiorse, S.R. (1993). SAMPE Quarterly, 24(2): 54-59.

18. Goodwin, A.A., Howe, C.A. and Paton, R.J. (1997). In: Proceedings of ICCM-11, Vol. 4, pp. 11-15.

19. Hoppel, C., Bogetti, T. and Newill, J.F. (2000). In: Proceedings of the American Society for Composites: 15th Technical Conference, pp. 1094-1101.

20. Varna, J., Joffe, R., Berglund, L. and Lundström, T. (1995). Composites Science and Technology, 53: $241-249$.

21. Wisnom, M.R., Reynolds, T. and Gwilliam, N. (1996). Composites Science and Technology, 56: 93-101.

22. Stabler, W.R., Tatterson, G.B., Sadler, R.L. and El-Shiekh, A.H.M. (1992). SAMPE Quarterly, 23(2): $38-42$.

23. Ikegawa, N., Hamada, H. and Maekawa, Z. (1996). Polymer Engineering and Science, 36(7): 953-962.

24. Russ, J. (1986). Practical Stereology, 1st Edn., Plenum Press, New York.

25. Ghiorse, S. (1991). U.S. Army Materials Technology Laboratory, Report MTL TR 91-13 pp. $1-20$.

26. Dhillon, I.S. (2000). Masters Thesis, University of Oklahoma, Norman, OK.

27. ASTM D3039/D3039M-95a (1997). 15.03. pp. 98-108.

28. Adams, K.L., Miller, B. and Rebenfeld, L. (1986). Polymer Engineering and Science, 26(20): 1434-1441.

29. Gauvin, R., Trochu, F., Lemenn, Y. and Diallo, L. (1996). Polymer Composites, 17(1): 34-42.

30. Patel, N., Rohatgi, V. and Lee, L.J. (1995). Polymer Engineering and Science, 35(10): 837-852. 